\title{
Éditorial
}

\section{Vers une nouvelle ruralité territorialisée}

Nos gouvernants aiment bien recourir à des commissions itinérantes pour sonder le pouls du bon peuple. $\mathrm{Au}$ moment d'écrire ces lignes, pas moins de trois Commissions parcouraient le Québec afin de connâ̂tre les points de vue susceptibles d'éclairer les politiques à venir. La plus célèbre portait sur les fameux accommodements raisonnables, une autre se penchait sur le sort réservé à nos aînés et enfin, celle se rapportant à l'avenir de l'agriculture et de l'agroalimentaire du Québec (CAAAQ) a publié son rapport au début de l'année. Notre participation à cette dernière sous la forme d'un mémoire présenté aux responsables de la MRC de Memphrémagog a inspiré l'idée d'un numéro spécial portant sur les défis de la ruralité, au Québec comme ailleurs, en ce début du XXI ${ }^{\mathrm{e}}$ siècle. Si elle fut moins médiatisée que la Commission visant à définir les grands traits de notre identité et les moyens à mettre de l'avant pour la préserver, la CAAAQ a soulevé un fort intérêt. Nous en voulons pour preuve les centaines de mémoires soumis à l'attention des commissaires.

Ça bouge dans les campagnes. Les changements sont parfois soudains et brutaux; c'est ce qui a fait dire à un observateur éclairé que Les belles histoires des pays d'en haut, Le Chenail du Moine et Le temps d'une paix appartiennent bel et bien à un autre monde. Les concepts d'agriculture durable et multifonctionnelle expriment de nouvelles préoccupations et territorialisent les actions qui s'y rattachent ${ }^{1}$. Ceci, pas seulement au pays du Québec, comme le montrent certains collaborateurs à ce numéro. Le sujet est dans le vent pour employer une expression de chez nous du début des années 1960. À preuve, le fait que La Revue d'économie régionale et urbaine a traité de la question à la faveur d'un numéro spécial : Les territoires du développement régional ${ }^{2}$. On y trouve une critique du modèle productiviste en agriculture et une description des problèmes environnementaux et des conflits qui l'accompagnent. Il y est fait allusion à la fonction écologique et paysagère de l'agriculture et de rôles nouveaux pour les agriculteurs. Ce qui nous renvoie à ce que l'OCDE qualifie de « nouveau paradigme rural $»^{3}$.

La nécessité de développer une vision nouvelle que l'on qualifie de nouvelles ruralités se trouve soulignée dans un dossier de Territoires $d u$ futur $^{4}$. Le concept fait appel à une prise de conscience nette et claire de l'existence de conflits dont l'acuité dans certains cas prend des dimensions grandissantes. On pense à la valeur foncière des sols agricoles qui s'accroît sous la pression des urbains argentés en mal de posséder un lieu de paix pour une retraite dorée. Ou encore le phénomène Pas dans ma cour! en ce qui regarde certaines activités (porcheries, éoliennes, etc.). Et que penser des exigences de nouveaux ruraux à la recherche de paysages bucoliques pour autant qu'ils y trouvent les services offerts en milieu urbain. On comprend les responsables de la revue Géographie, économie et société d'avoir consacré deux numéros aux Conflits d'usages et dynamiques spatiales: les antagonismes dans l'occupation des espaces périurbains et ruraux 5 . Nous sommes heureux à notre tour, de prendre part, à travers ce numéro, au débat en cours au pays des ancêtres de l'un des responsables de ce dossier.

Si l'on ne peut manquer de faire allusion à la multifonctionnalité de l'agriculture d'aujourd'hui, comme le signalent ici certains collaborateurs, c'est en fait de multifonctionnalité territoriale qu'il importe de parler, autant en ce qui regarde les activités productives que celles qui se rapportent à la préservation de l'environnement aux loisirs ou tout simplement aux lieux de vie. On pense ici à ce que notre collègue et amie Christel Alvergne qualifie dans son texte de nouvelles campagnes qui dégagent des dynamiques émergentes. Les pôles d'excellence régionale s'avèrent un exemple de ce qui est vu comme une entrée de plain-pied dans la nouvelle modernité sociétale et économique. Et comme le signalent de leur côté G. Domon et J. Ruiz, le territoire n'étant plus le support d'une fonction unique, il importe de substituer dans les plans d'action des approches territoriales aux traditionnelles 
approches sectorielles. Comment occuper le territoire ? Évidemment, en pensant à la relève, comme le démontre notre collègue de l'Université Laval D. Parent qui ne manque pas de croire en l'existence d'un dynamisme certain parmi nos jeunes. Et c'est aussi en pensant à l'approche territorialisée des systèmes agroalimentaires, comme le font nos collègues $F$. Jouve, C. Chazoule et R. Lambert, ce dernier étant également de l'Université Laval.

Cependant, il faut un cadre adéquat en agriculture comme pour tout autre activité. Ainsi, après avoir fait une remise en cause des structures administratives en place au Québec depuis le début des années 1980, comme le fait avec beaucoup de pertinence notre ami Y. Leclerc, D. Martel et J.-F. Albert, nous fournissent deux exemples de modèle d'occupation du territoire. Pour sa part, notre collègue uqamien L. Allie, avec l'exemple de nos Pactes ruraux, s'interroge si les responsables de la Politique nationale de la ruralité ne courent pas le risque de renforcer la logique administrative au détriment de la logique territoriale. Un rapprochement s'avère ici pertinent avec un exemple genevois mis en exergue par J. Ruegg qui signale des préoccupations ancrées ou territorialisées. C'est d'ailleurs à notre collègue helvète que nous devons le titre de ce dossier lorsque, en évoquant la question de la souveraineté alimentaire, il voit l'esquisse d'une nouvelle ruralité territorialisée.

Pour le Québec, avec les exemples fournis par notre collègue de l'INRS M. Simard, et nous-mêmes avec deux MRC qui se touchent, ou avec le cas de la Jamésie traité par nos amis de Niska à travers le concept de capital stratégique, on voit qu'il se fait des choses et qu'il pourrait s'en faire encore davantage. Il en va de même au Brésil, en France et en Acadie, comme le démontrent respectivement notre collègue Jandir Ferrera de Lima et notre ami Fabien Nadou. Oui, il pourrait se faire bien davantage, ici comme ailleurs. Pour le Québec, nous laissons le mot de la fin à Y. Leclerc qui considère qu'il faut retenir la division territoriale de la MRC comme territoire de développement local et définir les politiques de soutien aux acteurs à partir de ce milieu de vie.

Nos remerciements vont à tous ceux qui ont accepté de collaborer à ce numéro. Leur empressement à donner suite à notre invitation nous a conforté dans nos convictions sur l'intérêt d'un tel dossier.

$\begin{array}{ll}\text { André Joyal } & \text { KamalEl Batal } \\ \text { Université du Québec } & \text { Université du Québec } \\ \text { à Trois-Rivières } & \text { à Trois-Rivières }\end{array}$

Notes et références

1 Laurent, C. et P. Thinon (dir.) (2005). Agricultures et territoires, Paris, Lavoisier.

2 Revue d'économie régionale et urbaine, $\mathrm{n}^{\circ} 3$, 2007, p. 313520.

3 OCDE (2006). The New Rural Paradigme: Policies and Governance, Paris.

4 Dossier central : Les nouvelles ruralités. Aussi : J.C. Flamant, Var 2020, Quel futur pour l'agriculture?, mai 2007, $\mathrm{n}^{\circ} 6$.

5 Géographie, économie et société, vol 8, n 3, 2006 et vol. 9, nº 2. 2007. 